\title{
Electronic and Structural Characterization of Diamondoid Carbon Nanothreads by Transmission Electron Microscopy
}

Stephen Juhl ${ }^{1}$, Quentin Ramasse ${ }^{2}$, Xiang Li ${ }^{1}$, John Badding ${ }^{1,3}$, Nasim Alem ${ }^{3}$

1. Department of Chemistry, Pennsylvania State University, University Park, United States of America.

2. SuperSTEM Laboratory, STFC Daresbury Campus, Daresbury, United Kingdom.

3. Department of Materials Science and Engineering, Pennsylvania State University, University Park, United States of America.

Diamondoid carbon nanothreads are the first in a new category of one-dimensional $s p^{3}$-bonded carbon nanomaterials [1]. Recent studies have revealed single-crystalline packing of cylindrical structures into a two-dimensional pseudo-hexagonal lattice [1], [2]. These investigations reveal $\sim 20 \% s p^{2}$ bonding mixed in with the primarily diamond-like $s p^{3}$-bonded nanothreads [1]. While recent transmission electron microscopy (TEM) imaging reveals additional structural order at the nanoscale in this class of materials, the electronic and fine structure of diamondoid carbon nanothreads are completely unexplored and requires further investigation to elucidate its potential properties and illuminate the reaction mechanism [3], [4].

In this study, scanning/transmission electron microscopy (S/TEM) imaging coupled with electron energy-loss spectroscopy (EELS) is employed to examine the atomic, chemical, and electronic structure of carbon nanothreads. Low-dose EELS from the carbon K-edge in carbon nanomaterials reveals the bonding nature of the carbon to be primarily $s p^{3}$-bonded with hydrogen termination, as indicated by a small $\mathrm{C}=\mathrm{C} \pi^{*}$ peak $(284 \mathrm{eV})$, a broad C-C $\sigma^{*}$ peak $(290 \mathrm{eV})$, and the appearance of a C-H $\sigma^{*}$ peak $(287$ eV) (Figure 1a) [5]. Using S/TEM imaging and EELS this presentation will explore the beam-induced damage of the carbon nanothreads and the structural transformation of this nanomaterial as a result. Quantification of the $s p^{2} / s p^{3}$ ratio from our EELS measurements indicate that the nanothreads are primarily made of $s p^{3}$ bonded carbon atoms with a low $s p^{2}$ carbon content after accounting for the electron beam damage, as previously confirmed by NMR [1].

The electronic structure of the nanothreads is also probed by monochromated low-loss EELS to explore the present phonon vibrational modes in this crystal. The observed phonon modes appear at $170 \mathrm{meV}$ and $370 \mathrm{meV}$ which agree well with vibrational modes observed in Raman spectroscopy of bulk nanothreads (Figure 1b). In addition, this work will explore the optical band gap of the nanothreads and how it is compared with the existing calculations from ultraviolet-visible spectroscopy data [1]. Low loss EELS analysis as well as the plasmon peak also provide valuable insight on the bonding nature and the electronic structure of this nanostructure and is the key further explore potential applications of this unique carbon nanostructure.

References:

[1] T. C. Fitzgibbons et al, Nature Materials, 14 (2015), p. 43.

[2] X. Li et al, J. Am. Chem. Soc., 139 (2017) p. 16343.

[3] S. Juhl et al, Microsc. Microanal., 22 (2016) p. 1840.

[4] S. Juhl et al, Microsc. Microanal., 23 (2017), p. 1846.

[5] R. Brydson et al in "EMC 2008, Vol. 1", ed. M. Luysberg et al, (Springer-Verlag, Berlin Heidelberg) p. 357. 

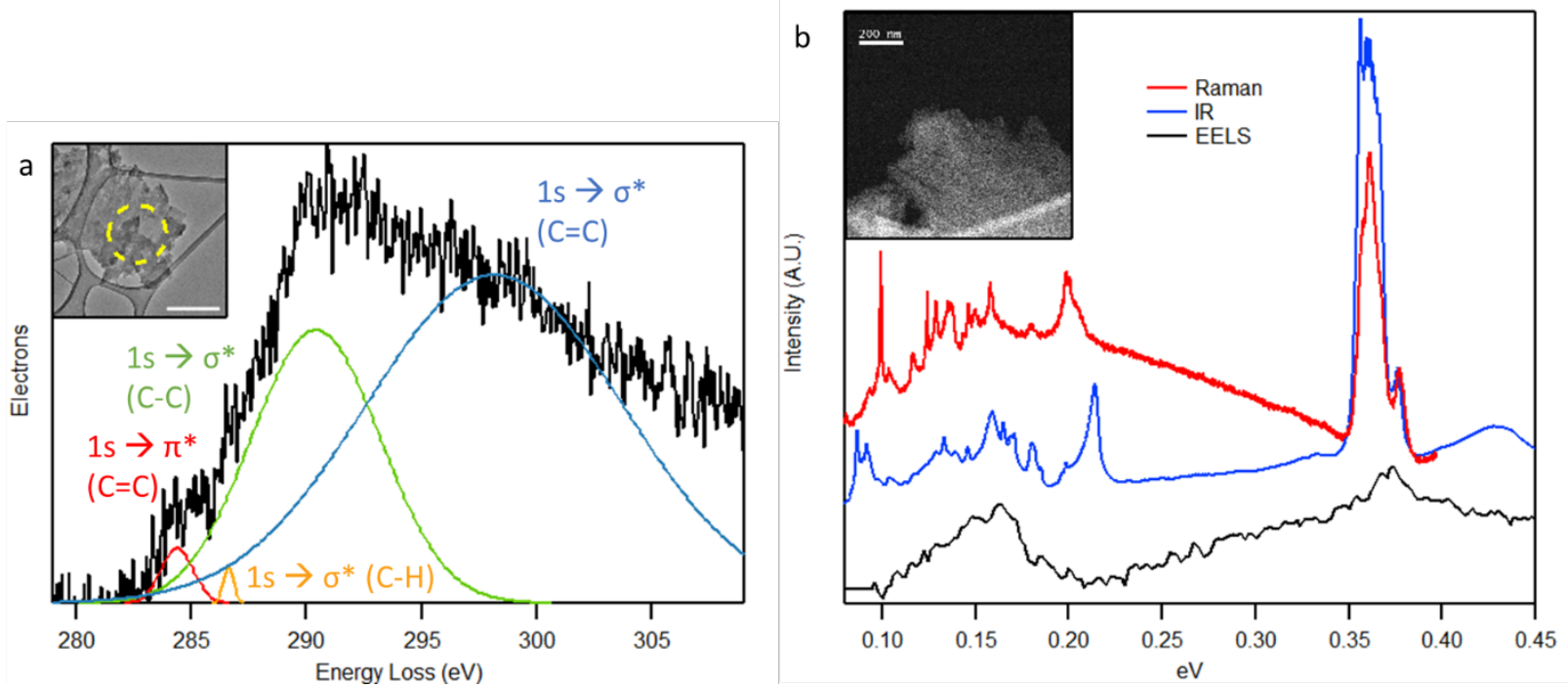

Figure 1. (a) Low-dose EELS spectrum of the carbon K-edge of carbon nanothreads with Gaussian fits of the major electronic transitions displayed. (b) Low-loss EELS spectrum of carbon nanothreads showing phonon modes compared with Raman and IR spectra of bulk nanothreads. 